\title{
Kernos
}

Revue internationale et pluridisciplinaire de religion grecque antique

$14 \mid 2001$

Varia

\section{Cl. CAlame, Poétique des mythes dans la Grèce antique}

\section{André Motte}

\section{OpenEdition}

Journals

Édition électronique

URL : http://journals.openedition.org/kernos/788

DOI : $10.4000 /$ kernos.788

ISSN : 2034-7871

Éditeur

Centre international d'étude de la religion grecque antique

Édition imprimée

Date de publication : 1 janvier 2001

Pagination : 315-316

ISSN : 0776-3824

Référence électronique

André Motte, "Cl. calame, Poétique des mythes dans la Grèce antique », Kernos [En ligne], 14 | 2001, mis en ligne le 14 avril 2011, consulté le 24 septembre 2020. URL : http://journals.openedition.org/ kernos/788; DOI : https://doi.org/10.4000/kernos.788 
regard critique qu'une discipline porte sur elle-mêrne et les stratégies mises en œuvre pour trouver la juste voie entre deux écueils : la généralisation réductrice et le relativisme radical...

Vinciane Pirenne-Delforge (FNRS - Université de Liège)

Claude Calame, Poétique des mytbes dans la Grèce antique, Paris, Hachette, 2000. 1 vol. $14 \times 22,5 \mathrm{~cm}, 287$ p. (Hachette Université. Langues et civilisations anciennes). ISBN : 2-01-021147-2.

Cet ouvrage, dont le titre éveille d'emblée l'intérêt, répond à une commande et est destiné prioritairement, comme le suggère déjà la collection dans laquelle il s'insère, à des étudiants désireux de s'initier à la culture hellène par la lecture des textes. Nul doute cependant qu'il ne retienne aussi l'attention des aînés. Les sept études ici produites, en effet, n'ont pas été sélectionnées et simplifiées ad usum delphini, si l'on peut dire, mais, d'une lecture exigeante, elles sont le fruit de recherches originales que l'A. a eu l'occasion déjà de présenter et de discuter au cours de séminaires ou de rencontres scientifiques. En outre, elles ne se bornent pas à expliquer des textes, mais elles mettent en œuvre toute une méthode de lecture dont les principes sont clairement énoncés, à la faveur notamment d'une critique mesurée de certaines approches traditionnelles de la mythologie grecque. L'énoncé des titres des chapitres ne fera qu'accroître l'appétit : «Bellérophon et la pragmatique du récit épique », "Clytemnestre et Oreste aux jeux pythiques », «Iô, les Danaïdes, l'extérieur et l'inflexion tragique », « Hélène et les desseins de l'historiographie », «Tirésias dans un hymne alexandrin », «Le panthéon de Trézène et Pausanias ». Cette dernière étude avait fait déjà l'objet d'une publication dans les Actes d'un colloque du C.I.E.R.G.A. (Les Panthéons des cités, Kernos, suppl. 8 [1998] p. 149-163); elle ici reprise et amplifiée. À noter que chaque chapitre est accompagné, en appendice, d'un ou deux textes anciens servant de base au commentaire, et de notes abondantes comportant de nombreuses références bibliographiques, la bibligraphie générale et finale se contentant, pour sa part, de reprendre une sélection d'ouvrages sur la mythologie grecque.

Il manque à cette énumération un chapitre, le premier, qui est de beaucoup le plus long (p, 11-60) et qui est sans doute aussi le plus important, car l'A. s'y emploie longuement à exposer sa «poétique » des mythes grecs. Son titre, "Créations narratives et poétiques ", dissimule le fait que le travail se fait, ici aussi, texte à l'appui, et ce n'est pas le moins captivant des textes retenus puisqu'il s'agit d'un long extrait du vieil Hymne bomérique à Déméter. Un choix s'imposant, c'est de ce chapitre introductif qu'on a choisi de donner un aperçu plus circonstancié. Mais disons d'abord un mot de l'Avant-propos qui commence par une sorte de manifeste, un "acte de foi ", comme il est dit. L'A. commence par prendre ses distances à l'égard de certaines analyses structurales qui, adoptant méritoirement une perspective comparative, sont restées trop braquées sur des éléments symboliques communs, gommant ainsi les différences et négligeant les contextes d'énonciation. Plaidant pour une approche consciente de ses présupposés et pour une rigueur méthodologique, il proteste, avec un agacement non dissimulé, contre un certain «néo-liberalisme de supermarché qui a désormais envahi les sciences humaines » et qui, préoccupé avant tout de succès médiatique, prône l'éclectisme et la concurrence individuelle, négligeant du même coup les questions de méthode et de cohérence épistémologique.

Les créations narratives et poétiques des Grecs méritent mieux que cela! Reprenant des idées qu'il a déjà eu l'occasion d'exposer ailleurs, mais dont il précise ici certains points et qu'il va illustrer à nouveau dans diverses applica- 
tions, $\mathrm{Cl}$. Calame aborde son premier chapitre par une réflexion sur la notion de mythe, montrant que mutbos, à l'époque archaïque et classique, ne désigne pas une classe de récits traditionnels auxquels on dénierait toute valeur de vérité. Le muthos ne s'oppose pas ici à un quelconque logos. S'il arrive que des philosophes critiquent des mythes, c'est en raison de la fonction éducative que ces récits poétiques exercent dans la cité et que parfois, à leurs yeux, ils assument dans un sens qui ne convient pas. Les mythes grecs n'ont d'existence que dans des compositions poétiques qui les portent à leur public. On doit regretter, d'autre part, que l'anthropologie ait accrédité la notion de mythe comme mode particulier de la pensée humaine, - une réalité transcendentale en quelque sorte -, la délestant ainsi de son aspect narratif et appauvrissant du même coup la richesse sémantique de ces formes de discours toujours liés à des manifestations particulières de la vie sociale et culturelle. Une impressionnante revue des interprétations qu'a connues, depuis l'Antiquité jusqu'à nos jours, le mythe de Déméter et de Corè, révèle une tendance commune à faire des mythes des universaux, en même temps qu'elle conduit à relativiser ces essais successifs de naturalisation et d'ontologisation de catégories interprétatives. En réalité, " chaque mythe ne peut être lu que dans la version particulière qui le porte à son destinataire ". C'est ce que l'A. va tenter de faire un peu plus loin à propos du rapt de Perséphone tel que le raconte l'Hymne bomérique : cette trame narrative doit être comprise dans le complexe d'effets de sens qu'elle suscite et dans l'accomplissement du rituel que le récit est censé instituer. Auparavant, il aura montré, en scrutant les dits de plusieurs penseurs grecs, que les Anciens ont toujours été sensibles à la valeur pragmatique des récits fictionnels.

Ces trop brèves esquisses auront fait, je l'espère, pressentir les mérites de cet ouvrage dont il faut souhaiter qu'il suscite un large débat.

André Motte (Université de Liège)

Frontisi-Ducroux Françoise, Dédale. Mythologie de l'artisan en Grèce ancienne. Préface de Pierre Vidal-Naquet. Posface inédite de l'auteur, Paris, La Découverte/Poche, 2000. 1 vol. $12,5 \times 19 \mathrm{~cm}, 241$ p. (Sciences bumaines et sociales). ISBN : 2-7071-3311-6.

Ce petit ouvrage est la réimpression anastatique de la thèse de l'A. initialement publiée en 1975, à laquelle est ajoutée une postface intitulée Dédale, an 2000. Des daidala à la figure de Dédale, c'est tout le champ de la mètis artisanale qu'explore cet ouvrage, que rien n'est vraiment venu remplacer depuis sa publication, pas même le Daidalos de Sarah Morris (Princeton, 1992) conçu dans une perspective toute différente. On saura gré à l'A. de mettre clairement en évidence ce qu'elle n'aurait pas refait à l'identique, s'il avait fallu réécrire ce livre, et le pourquoi de ces « repentirs ». Il s'agit essentiellement des développements qui concernent les relations entre le mythe de Dédale, les débuts de la sculpture grecque et la technologie du bronze, en somme tout ce qui pose le problème « du rapport de la création mythique avec les réalités techniques » (p. 220). Les représentations grecques de l'origine de la sculpture relèvent de la construction intellectuelle plus que de l'histoire de l'art proprement dite. Elles relèvent autant du mythe que le personnage de Dédale lui-même. Mais il est impossible de s'en tenir à ce seul constat. Et l'A. d'affirmer quelques pages plus loin : « Le problème demeure cependant du rapport des constructions de l'imaginaire avec la réalité. Il s'agit de savoir ce qui dans le réel a été effectivement sélectionné, ce qui a stimulé la création mythique, poétique ou figurative », tous ces « objets bons à penser » chers à Lévi-Strauss (p. 224). La postface ouvre donc de nouvelles 